04

\title{
Влияние предыонизации на микроканальную структуру искрового разряда в воздухе в промежутке острие-плоскость
}

\author{
(ㄷ А.А. Тренькин, К.И. Алмазова, А.Н. Белоногов, В.В. Боровков, Е.В. Горелов, \\ И.В. Морозов, С.Ю. Харитонов
}

Российский Федеральный ядерный центр

Всероссийский научно-исследовательский институт экспериментальной физики, 607190 Саров, Россия

e-mail: alexey.trenkin@gmail.com

Поступило в Редакцию 12 августа 2021 г.

В окончательной редакции 4 октября 2021 г.

Принято к публикации 5 октября 2021 г.

Методом теневого фотографирования исследована начальная фаза искрового разряда в промежутке острие (катод)-плоскость длиной $1.5 \mathrm{~mm}$ в воздухе атмосферного давления в условиях предварительной фотоионизации вспомогательным разрядом. В отсутствие предыонизации разряд с первых наносекунд после пробоя представляет собой совокупность большого числа каналов микронного диаметра. Установлено, что реализующаяся в результате предыонизации концентрация электронов, оцениваемая на уровне $10^{8}-10^{9} \mathrm{~cm}^{-3}$, повышает степень однородности канала разряда в прикатодной области, однако в прианодной области канал продолжает оставаться микроструктурированным. В рамках механизма формирования микроструктуры за счет неустойчивости фронта волны ионизации получен критерий формирования однородного разряда и представлено объяснение полученных результатов.

Ключевые слова: искровой разряд, предыонизация, фотоионизация, неустойчивость фронта ионизации, микроструктура, метод теневого фотографирования.

DOI: 10.21883/JTF.2022.01.51851.237-21

\section{Введение}

Исследование влияния предварительной ионизации на динамику развития газовых разрядов весьма актуально, например, для создания газовых лазеров, в плазмохимических технологиях и т.д. Как правило, основной целью предыонизации является создание достаточно однородного разряда в требуемом объеме в некотором интервале времени.

Проблеме формирования однородных разрядов в больших объемах и при высоких давлениях (прядка $1 \mathrm{~atm}$ и выше) посвящено большое количество работ (см., например, обзор [1] и ссылки в нем). Полагается, что условием однородного развития разряда является пространственное перекрытие соседних электронных лавин до достижения ими критического размера при условии их равномерного распределения по рабочему объему $[1,2]$. Критическим радиусом $r_{\text {сr }}$ считается радиус головки лавины в момент лавинно-стримерного перехода, а равномерность распределения точек инициирования лавин по рабочему объему обеспечивается внешней предыонизацией, в результате чего создается начальная концентрация электронов, которая должна составлять $n_{0}>r_{\mathrm{cr}}^{-3}=10^{4} \mathrm{~cm}^{-3}[1,3]$. Дальнейшее развитие данной модели потребовало для критерия зажигания объемного разряда наличия в промежутке начальных электронов с концентрацией $n_{0}>10^{7} \mathrm{~cm}^{-3}$, что лучше соответствовало экспериментальным данным [1]. Такое условие на начальной стадии развития разряда должно предотвращать формирование отдельных токовых нитей диаметром порядка $0.1 \mathrm{~cm}$ с последующим контрагированием разряда.

Вместе с тем в ряде работ [4-10] было установлено, что внешне выглядящие однородными разряды могут обладать внутренней микроструктурой - реализовываться в виде совокупности большого числа микроканалов. При этом оптическими и электронно-оптическими методами микроструктура была не разрешима [4].

Микроструктура в объеме разрядного промежутка была зарегистрирована относительно недавно с применением метода лазерного зондирования и основанных на нем теневых и интерференционных методиках [4-7]. В качестве одного из наиболее приемлемых объяснений данного явления рассматривается механизм формирования микрокананалов в результате неустойчивости фронта ионизации в начальной фазе разряда [9-15].

Микроструктура зарегистрирована в различных типах газовых разрядов [4-10]. В этой связи весьма интересным и практически важным является вопрос о влиянии предыонизации на пространственную структуру таких разрядов.

Настоящая работа является продолжением исследований $[4,5]$, однако в отличие от них здесь использован двухкадровый вариант методики теневого фотографирования, что значительно расширяет возможности получения данных о процессах, сопровождающих газовые разряды. В настоящей работе представлены результаты исследования влияния предыонизации на микроканальную 
структуру искрового разряда в воздухе в промежутке острие-плоскость на начальной стадии его развития.

\section{1. Экспериментальная аппаратура и методика}

Схема экспериментального стенда представлена на рис. 1. Большая часть элементов схемы подробно описана в $[4,5]$. Генераторы импульсов напряжения (ГИН) обеспечивали на выходе импульс напряжения амплитудой $25 \mathrm{kV}$ и длительностью фронта по уровню $0.1-0.9$ около $7 \mathrm{~ns}$. Через кабельные линии импульсы подавались на разрядные промежутки. Один генератор (ГИН 1) обеспечивал формирование основного (исследуемого) разряда, другой (ГИН 2) - вспомогательного, реализующего предварительную фотоионизацию воздуха для основного разряда. Пуски ГИН осуществлялись с заданной задержкой. Длина каждой кабельной линии для питания основного и вспомогательного разрядов составляла $7 \mathrm{~m}$.

Электродная система для формирования основного разряда имела геометрию „острие-плоскость“. Осесимметричный острийный электрод был изготовлен из нержавеющей стали и имел длину $19 \mathrm{~mm}$, диаметр $14 \mathrm{~mm}$, угол при вершине $36^{\circ}$ и радиус кривизны $0.15 \mathrm{~mm}$. В качестве плоского электрода использовался электрод, изготовленный из алюминиевого сплава, с рабочей частью, близкой по форме к шаровому сегменту диаметром $4.5 \mathrm{~cm}$ толщиной $1.5 \mathrm{~cm}$. Межэлектродный зазор составлял $1.5 \mathrm{~mm}$.

Вспомогательный разряд формировался между жилой и оплеткой на конце кабеля, срезанного перпендикулярно оси. Плоскость среза располагалась параллельно оси основного разрядного промежутка на расстоянии $1 \mathrm{~cm}$ от нее.

Для основного разряда измерялись напряжение $U$ и ток $I$, для вспомогательного - только ток $I_{i}$. Измерения осуществлялись на выходе ГИН. Для измерения напряжения и тока использовался соответственно емкостной делитель и резистивный шунт. Временное разрешение делителя и шунта - не хуже $1 \mathrm{~ns}$. Регистрация сигналов производилась осциллографом с полосой пропускания $500 \mathrm{MHz}$ и скоростью оцифровки $2 \mathrm{Gs} / \mathrm{s}$.

Для исследования основного разряда использовалась система оптической регистрации. Система включала в себя источник зондирующего излучения - твердотельный лазер (длина волны $532 \mathrm{~nm}$, длительность импульса на полувысоте $6 \mathrm{~ns}$ ), объективы, светофильтры и цифровые электронно-оптические регистраторы. Плоскопараллельный пучок лазерного излучения, проходя через область разряда перпендикулярно оси электрода-острия, регистрировался электронно-оптической камерой. В области формирования разряда поперечный размер лазерного пучка составлял примерно $1 \mathrm{~cm}$ и имел гауссов профиль.

На базе этой системы реализована методика теневого фотографирования, в которой использовалась двулуче-

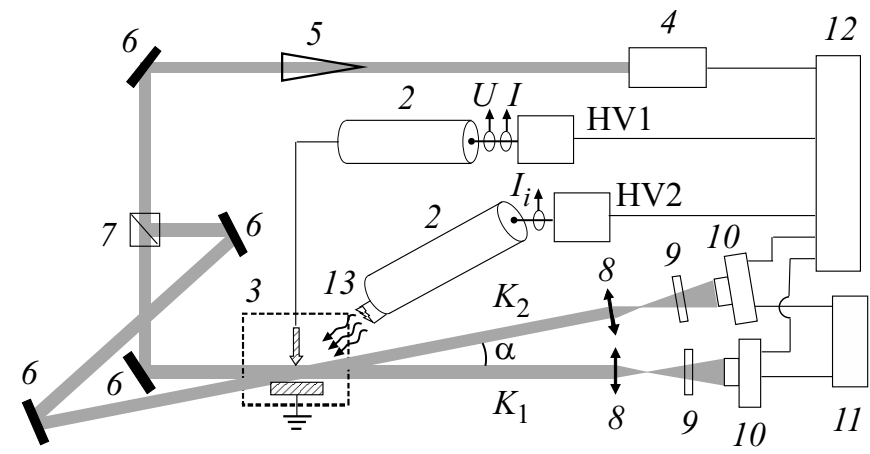

Pис. 1. Схема экспериментального стенда. HV1 и HV2 - генераторы импульсов напряжения, 2 - кабельная линия, 3 - разрядный промежуток, $4-$ источник зондирующего сигнала (лазер), 5 - коллиматор, $6-$ поворотное зеркало, 7 - светоделительный элемент, 8 - объектив, 9 - светофильтры, 10 - электронно-оптический регистратор, 11 - персональный компьютер, 12 - блок синхронизации, 13 - вспомогательный разряд, осуществляющий предыонизацию разрядного промежутка.

вая оптическая схема, что обеспечивало получение двух кадров за импульс (рис. 1). Угол $\alpha$ между лазерными лучами составлял около $15^{\circ}$. Для каждого луча изображение в области разрядного промежутка строилось с помощью объектива с фокусным расстоянием $23 \mathrm{~cm}$ на фотокатоде электронно-оптического регистратора. Коэффициент увеличения равен десяти. Экспозиция каждого кадра определялась длительностью импульса лазера. Изменением разности оптической длины пути лучей $K_{1}$ и $K_{2}$ можно было варьировать временной интервал между кадрами тенеграмм. В настоящих экспериментах этот интервал составлял $5 \mathrm{~ns}$. Временная привязка кадров осуществлялась относительно момента пробоя, а характеризующее их время соответствовала началу кадра.

Сдвигом момента запуска лазера и электроннооптического регистратора относительно момента пробоя обеспечивалась визуализация различных стадий разрядного процесса. Разрешающая способность оптической системы составляла $5 \mu \mathrm{m}$ на три пикселя.

Интегральное свечение вспомогательного разряда фотографировалось цифровым фотоаппаратом.

\section{2. Экспериментальные результаты и их обсуждение}

Опыты проводились в воздухе при нормальных условиях. В отсутствие предыонизации данный разряд был достаточно подробно исследован в работах $[4,5]$.

Использовались две редакции экспериментов: с предыонизацией разрядного промежутка и без нее. В первом случае разряды синхронизировались таким образом, чтобы пробой основного промежутка происходил в максимуме тока вспомогательного разряда. Фрагменты осцил- 


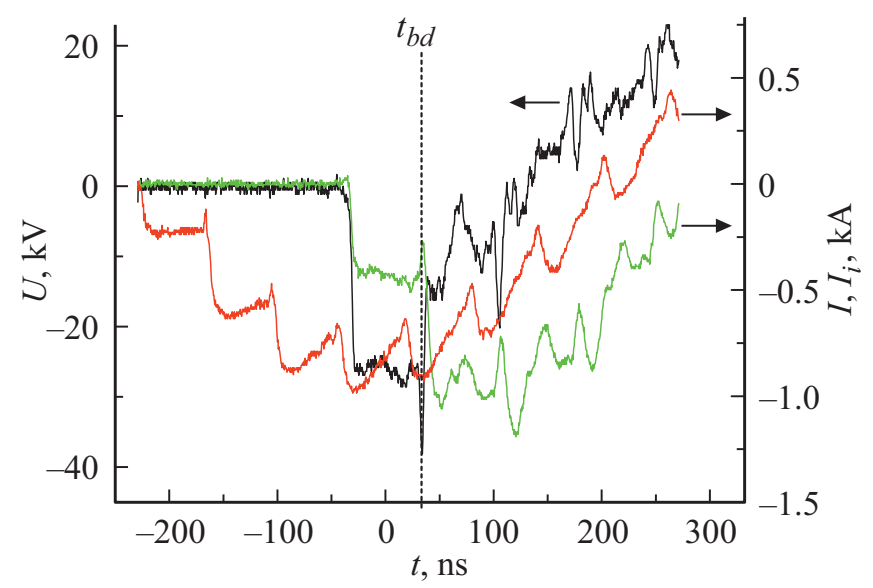

Рис. 2. Осциллограммы тока $I$ (зеленая линия (в онлайн версии)) и напряжения $U$ (черная линия) на выходе ГИН 1 , а также тока $I_{i}$ (красная линия (в онлайн версии)) на выходе ГИН 2, $t_{b d}$ - момент пробоя.

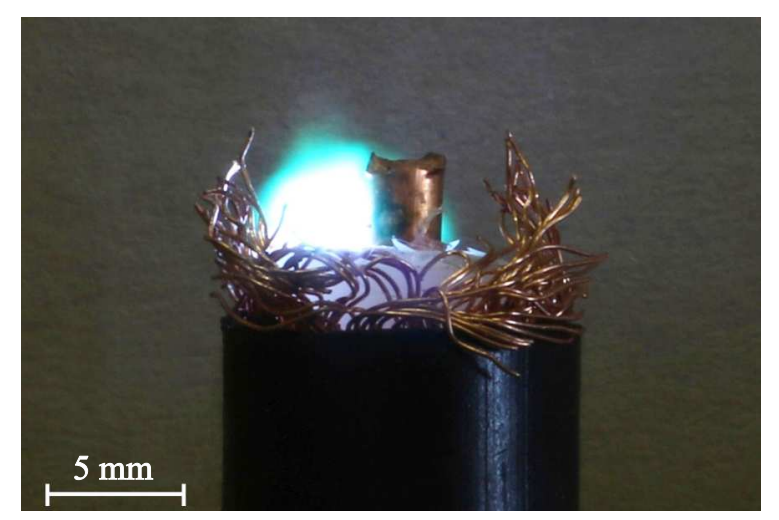

Рис. 3. Фотография вспомогательного разряда.

лограмм тока и напряжения на выходе ГИН 1, а также тока на выходе ГИН 2 приведены на рис. 2. После пробоя основного промежутка в разрядной цепи возникал колебательный процесс с экспоненциальным затуханием тока и напряжения. Период колебаний составлял $0.6 \mu \mathrm{s}$, амплитуда тока и время его затухания, соответственно, $1 \mathrm{kA}$ и $1.2 \mu \mathrm{s}$. За момент пробоя был принят момент начала роста тока и соответственно спада напряжения. Наличие предыонизации не оказало заметного влияния на осциллограммы основного разряда. Амплитуда тока вспомогательного разряда составляла около $1 \mathrm{kA}$. Его интегральное свечение представлено на рис. 3.

На рис. 4 представлены тенеграммы разряда в отсутствие предыонизации. Видно, что канал разряда представляет собой совокупность большого числа микроканалов. Следует отметить, что от импульса к импульсу структура канала отличалась по степени однородности в прикатодной области. Так, на рис. $4, a, b$ микроструктура присутствует, в том числе и в прикатодной области. В другом импульсе (рис. $4, c, d$ ) на тенеграммах, соответ- ствующих примерно тем же временам, участок канала в прикатодной области выглядит более однородным, микроканалы здесь не разрешимы. Отметим, что об однородном (бесструктурном) характере канала вблизи острийного катода сообщалось в работе [7].

На рис. 5 представлены тенеграммы разряда в случае предварительной фотоионизации промежутка вспомогательным искровым разрядом. Видно, что на участке от катода и примерно до половины длины промежутка канал является однородным, без микроструктуры. В оставшейся части присутствует микроканальная структура, которая сохраняется и на более поздних стадиях. Такая картина наблюдалась на времена до 15-20 ns после пробоя во всех импульсах. Укажем, что в ряде случаев на более поздних стадиях микроструктура наблюдалась во всем канале, включая прикатодную область. Следует отметить, что в это время внешний источник ионизации продолжал работать.

Таким образом, влияние предыонизации заключается в повышении степени однородности канала в начальной стадии разряда. При этом реализуется двуфазная структура: канал состоит из бесструктурного участка, контактирующего с катодом, и микроструктурированного участка, контактирующего с анодом. В целом эксперименты показывают, что используемый источник предыонизации не обеспечивает формирование однородного разряда во всем промежутке.

Рассмотрим механизм влияния предварительной ионизации на микроструктуру разряда в рамках модели ее формирования за счет неустойчивости ионизационного фронта [9,10]. Согласно этой модели, при определенных условиях ионизационный фронт может быть неустойчивым по отношению к пространственным возмущениям электронной концентрации в определенном диапазоне их размера [9-15]. Развитие неустойчивости приводит к возникновению микроканалов. Очевидно, что для подавления такой неустойчивости характерное расстояние между электронами, возникающими за счет предыонизации, не должно превышать характерного размера неоднородности, имеющей положительный инкремент роста. Полагая, что радиус такой неоднородности соответствует радиусу $r_{\text {inst }}$ микроканалов в начальной стадии разряда, для регистрируемых значений порядка $r_{\text {inst }}=10 \mu \mathrm{m}$, имеем оценку начальной концентрации электронов $n_{0}>r_{\text {inst }}^{-3}=10^{9} \mathrm{~cm}^{-3}$.

На основе данных $[16,17]$ по исследованиям искрового разряда как источника фотоионизации с параметрами, близкими к используемым в настоящей работе, можно оценить концентрацию электронов, обеспечиваемую таким источником.

Так, согласно измерениям для разряда в воздухе атмосферного давления [16], средняя мощность излучения $w$ в ультрафиолетовом диапазоне составляла около $2 \mathrm{~mJ} /\left(\mathrm{cm}^{2} \cdot \mathrm{s}\right)$ на расстоянии $1 \mathrm{~cm}$ от разряда. Концентрацию электронов, реализуемую при фотоионизации в области канала разряда длиной $l=1.5 \mathrm{~mm}$ и радиусом 

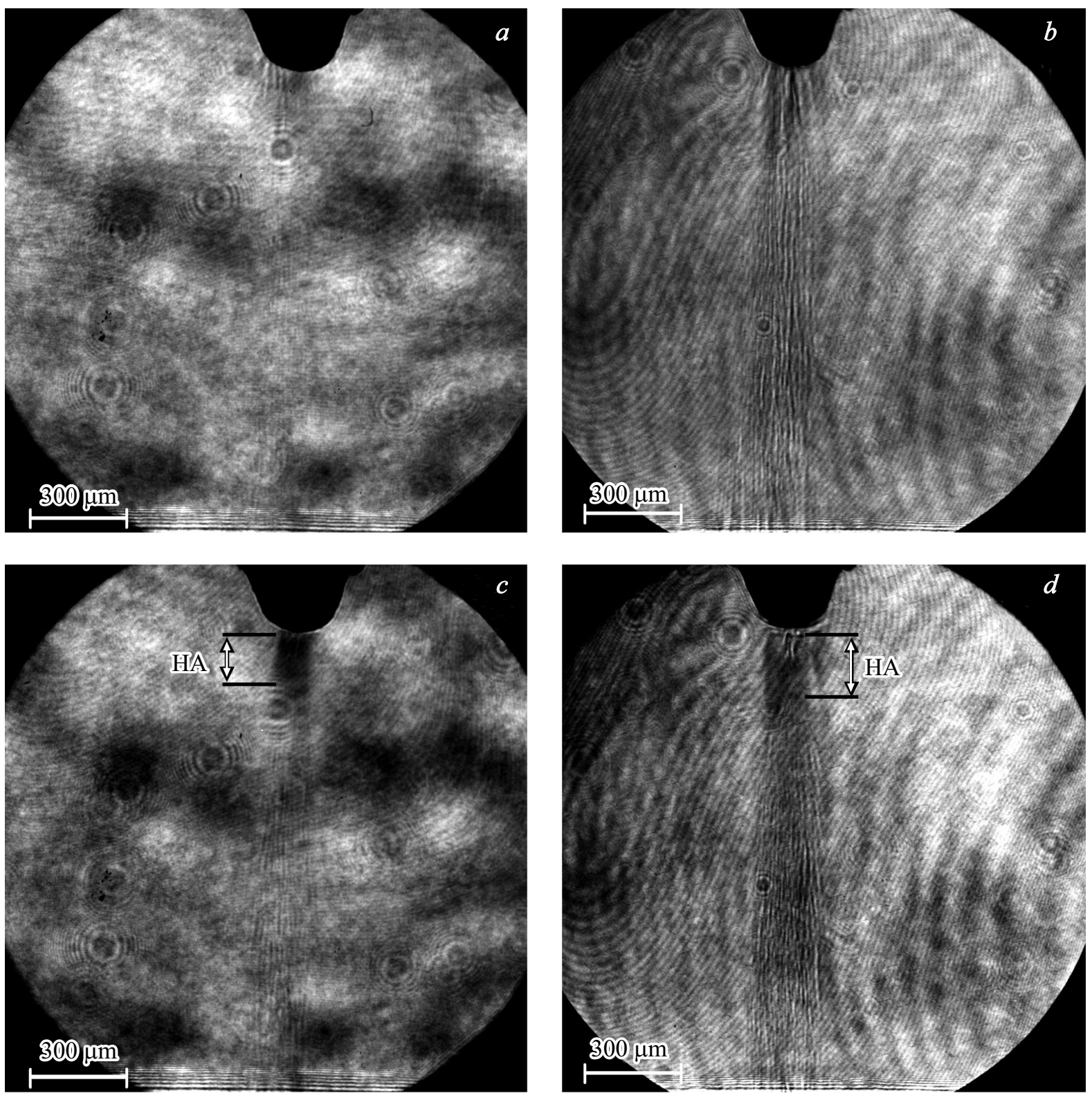

Рис. 4. Тенеграммы разряда без предыонизации. Пары изображений $(a, b)$ и $(c, d)$ получены в разных импульсах. Время относительно пробоя: $a-9, b-14, c-10, d-15 \mathrm{~ns}$. НА - область с повышенной степенью однородности.

$r=150 \mu \mathrm{m}$ можно оценить как

$$
n_{e}=w S_{\mathrm{ch}} \Delta t /\left(W_{i} V_{\mathrm{ch}}\right) \text {. }
$$

Полагая $W_{i}=14.5 \mathrm{eV}$ - потенциал ионизации азота, $\Delta t=100 \mathrm{~ns}$ - характерная лительность воздействия источника ионизации, $S_{\mathrm{ch}}=2 r l-$ площадь сечения канала вдоль его оси, $V_{\mathrm{ch}}=\pi r^{2} l-$ объем канала, получаем $n_{e} \approx 10^{10} \mathrm{~cm}^{-3}$. Полученное значение, повидимому, следует рассматривать как завышенное ввиду используемого здесь идеализированного предположения о стопроцентной эффективности фотоионизации ультрафиолетовым излучением.

В работе [17] был использован расчетно-экспериментальный подход к определению концентрации электро- нов, реализуемой при фотоионизации азота атмосферного давления, который дает $n_{e} \approx 5 \cdot 10^{7} \mathrm{~cm}^{-3}$ на расстоянии $8 \mathrm{~cm}$ от источника в момент достижения током разряда амплитудного значения. Следует ожидать, что на расстоянии $1 \mathrm{~cm}$ от источника концентрация электронов будет выше. При этом представляется обоснованным перенос полученных результатов с азота на воздух при условии использования рассматриваемых здесь относительно мощных источников фотоионизации в течение времени их работы.

Таким образом, можно полагать, что реализуемая в настоящих экспериментах предварительная ионизация промежутка вспомогательным искровым разрядом 

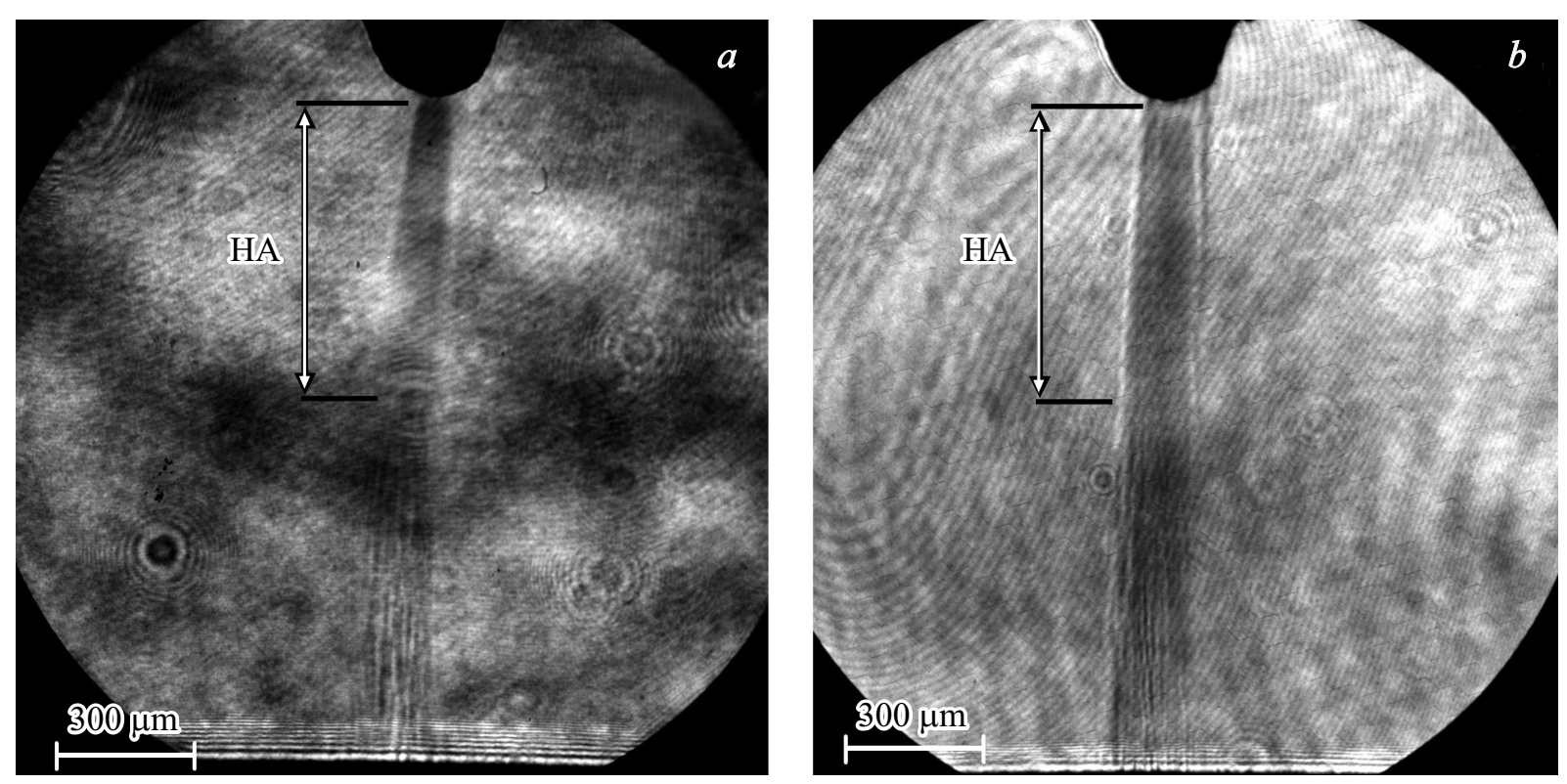

Рис. 5. Тенеграммы разряда с предыонизацией. Изображения получены в одном импульсе. Время относительно пробоя: $a-7$, $b-12$ ns. НА - область с повышенной степенью однородности.

обеспечивает концентрацию электронов $n_{e}$ в диапазоне $10^{8}-10^{9} \mathrm{~cm}^{-3}$.

Полученные оценочные значения свидетельствуют о том, что реализуемая за счет предыонизации концентрация электронов не достаточна для формирования однородного канала во всем промежутке. Вместе с тем в прикатодной области необходимо учитывать наличие относительно высокого электрического поля, обусловливающего эмиссионные и ионизационные процессы и соответственно повышенный фон электронов. В этом случае вклад внешней предыонизации, по-видимому, способен обеспечить формирование однородного участка канала вплоть до примерно середины разрядного промежутка.

Тот факт, что однородный участок канала разряда в прикатодной области зачастую наблюдался и без внешней предыонизации, по-видимому, также связан с интенсивными ионизационными процессами в этой области, определяющими в том числе особенности развития ионизационной неустойчивости. Подробно динамика указанных процессов рассмотрена в [10].

На основании полученных результатов можно сделать следующие выводы. Во-первых, ожидается, что внешний источник предыонизации большей мощности $\left(n_{e} \gg n_{0}\right)$ по сравнению с использованным в настоящей работе обеспечит формирование однородного разряда во всем промежутке. Во-вторых, если разряд обладает микроструктурой, то необходимые для реализации однородного разряда минимальные концентрации электронов предыонизации могут значительно превышать ранее принятые значения $[1,3]$.

\section{Заключение}

С использованием двухкадровой методики теневого фотографирования исследовано влияние предыонизации излучением от вспомогательного разряда на динамику развития начальной стадии искрового разряда в промежутке острие (катод)-плоскость длиной $1.5 \mathrm{~mm}$ в воздухе атмосферного давления.

Показано, что в отсутствие предыонизации с первых наносекунд после пробоя канал обладает пространственной микроструктурой - представляет собой совокупность большого числа микроканалов. При этом от импульса к импульсу участок канала в прикатодной области был либо микроструктурирован, либо однороден.

Установлено, что влияние предыонизации в начальной фазе разряда заключается в повышении степени однородности канала разряда: канал состоит из бесструктурного участка от катода до, примерно, середины промежутка и обладает микроструктурой в прианодной области.

Высказано предположение о том, что повышение степени однородности при предыонизации происходит за счет подавления неустойчивости фронта волны ионизации, ответственной за формирование микроструктуры. Получена оценка критического значения концентрации электронов предыонизации, при превышении которой ионизационная неустойчивость не развивается. Данное значение (порядка $10^{9} \mathrm{~cm}^{-3}$ ) оказалось близко к концентрации электронов предыонизации, оцениваемой на уровне $10^{8}-10^{9} \mathrm{~cm}^{-3}$.

Ожидается, что внешний источник предыонизации большей мощности, реализующий концентрацию электронов, значительно превосходящую критическую, обес- 
печит формирование однородного разряда во всем промежутке.

Отмечено, что для обладающих микроструктурой разрядов необходимая для реализации однородного разряда минимальная концентрация электронов предыонизации может значительно превышать ранее принятые значения $10^{4}-10^{7} \mathrm{~cm}^{-3}[1,3]$.

\section{Конфликт интересов}

Авторы заявляют, что у них нет конфликта интересов.

\section{Список литературы}

[1] В.В. Осипов. УФН, 170 (3), 225 (2000).

[2] В.Н. Карнюшин, Р.И. Солоухин. ДАН СССР, 236 (2), 347 (1977).

[3] Э.М. Базелян, Ю.П. Райзер. Искровой разряд (МФТИ, М., 1997)

[4] К.И. Алмазова, А.Н. Белоногов, В.В. Боровков, Е.В. Горелов, И.В. Морозов, А.А. Тренькин, С.Ю. Харитонов. ЖТФ, 88 (6), 827 (2018). DOI: 10.21883/JTF.2018.06.46011.2511 [K.I. Almazova, A.N. Belonogov, V.V. Borovkov, E.V. Gorelov, I.V. Morozov, A.A. Trenkin, S.Yu. Kharitonov. Tech. Phys., 63 (6), 801 (2018). DOI: 10.1134/S1063784218060026]

[5] А.А. Тренькин, К.И. Алмазова, А.Н. Белоногов, В.В. Боровков, Е.В. Горелов, И.В. Морозов, С.Ю. Харитонов. ЖТФ, 90 (12), 2039 (2020). DOI: $10.21883 /$ JTF.2020.12.50119.435-19 [A.A. Trenkin, K.I. Almazova, A.N. Belonogov, V.V. Borovkov, E.V. Gorelov, I.V. Morozov, S.Yu. Kharitonov. Tech. Phys., 65 (12), 1948 (2020). DOI: 10.1134/S1063784220120270]

[6] E.V. Parkevich, M.A. Medvedev, A.I. Khirianova, G.V. Ivanenkov, A.S. Selyukov, A.V. Agafonov, K.V. Shpakov, A.V. Oginov. Plasma Sources Sci. Technol., 28, 125007 (2019). DOI: 10.1088/1361-6595/ab518e

[7] E.V. Parkevich, M.A. Medvedev, G.V. Ivanenkov, A.I. Khirianova, A.S. Selyukov, A.V. Agafonov, Ph.A. Korneev, S.Y. Gus'kov, A.R. Mingaleev. Plasma Sources Sci. Technol., 28, 095003 (2019). DOI: 10.1088/1361-6595/ab3768

[8] А.Г. Репьев, П.Б. Репин, В.С. Покровский. ЖТФ, 77 (1), 56 (2007). [A.G. Rep'ev, P.B. Repin, V.S. Pokrovski'. Tech. Phys., 52 (1), 52 (2007).]

[9] А.А. Тренькин, В.И. Карелин. ЖТФ, 78 (3), 29 (2008). [A.A. Trenkin, V.I. Karelin. Technical Physics, 53 (3), 314 (2008). DOI: 10.1134/S1063784208030055]

[10] В.И. Карелин, А.А. Тренькин. ЖТФ, 78 (9), 134 (2008). [V.I. Karelin, A.A. Trenkin. Tech. Phys., 53 (9), 1236 (2008). DOI: $10.1134 / \mathrm{S} 106378420809017 \mathrm{X}]$

[11] Э.Д. Лозанский, О.Б. Фирсов. Теория искры (Атомиздат, М., 1975)

[12] О.А. Синкевич. ТВТ, 41 (5), 695 (2003).

[13] M. Arrayas, M. Fontelos, J. Trueba. Phys. Rev. Lett., 95 (5), 165001 (2005). DOI: 10.1103/PhysRevLett.95.165001

[14] A. Rocco, U. Ebert, W. Hundsdorfer. Phys. Rev. E, 66, 035102(R) (2002). DOI: 10.1103/PhysRevE.66.035102

[15] A. Luque, F. Brau, U. Eber. Phys. Rev. E, 78, 016206 (2008). DOI: 10.1103/PhysRevE.78.016206

[16] I.M. Piskarev, I.P. Ivanova, S.V. Trofimova. Intern. J. Appl. Fundament. Res., 60, 12 (2014).

[17] Е.И. Козлова, А.Ю. Колесников, А.А. Котов, В.П. Новиков. ФП, 32 (5), 477 (2006). 